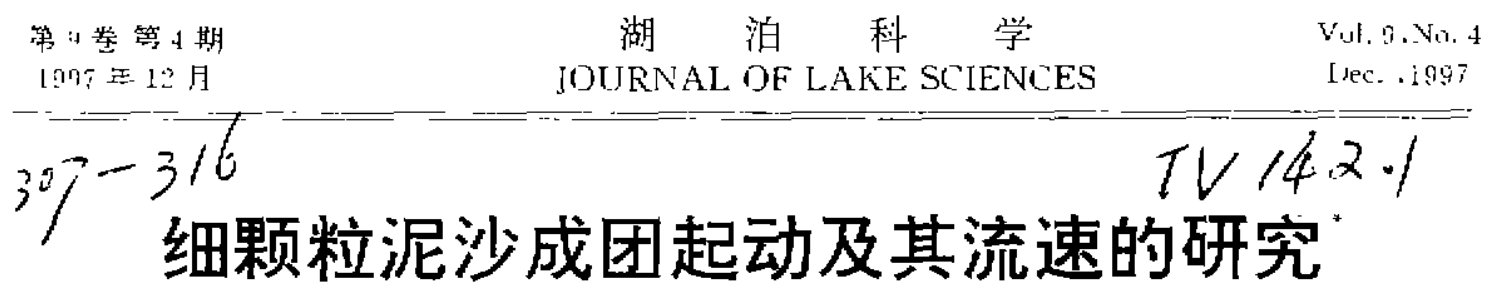

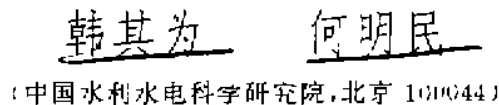

\begin{abstract}
提要文中首先指出当水菻很大时.考㽽薄膜水附加下压力的起动流速公式计算的单颗细

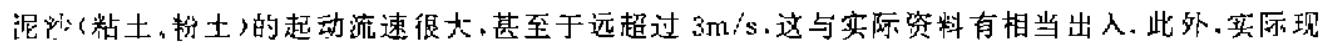
象还表明这些粘土及细颗精起动时常常不是单颗的, 而是成团进行. 由此出发.再考虑粘着力及薄 膜水附乐力下分析?土块成团起动的临界条件,得到了成团起动的起动流速. 同时对比了成团(包 括成片，起动流速与单颗的起动流速，得出了临界水深 $H_{K}$. 当 $H=H_{K}$ 时，成圈起动流速等于单影 的：当 $H<K_{K}$ 时成团起动流速大于单颗的，当 $H>H_{K}$ 时成团起动流速小于单颗的. 在后一仲条 件下.将以成团起动为主. 最后通过一些数据显示，对于粘土、精沙， $H_{K}$ 随团块的当量粒空 $D_{3}$ 瑊 小、干容重瑊小、土块扁度加大、单颗泥沙直径减小两减小, 同时在一定条件下 $H_{K}$ 可以很小。已至 为零: 这表明成块起动可能发生在任何水浑(包括水槽试验中?
\end{abstract}

关链词成图起动 细颗精临界水深片状土块，

单颗细沙起动研究中.除水深通讨流速分布影响起动流速外, 是否还存在其它与水深有关 的影响存在争议。一种认为不存在其它影响 ${ }^{[1]}$; 另一种认为由于薄膜水单向压力传递. 水深对 薄膜水附加下匤力有影响而牵连到起动流速 ${ }^{-2-4]}$; 第三种认为除流速分布影响外, 尚有细颗粒 处于枮性于层而阻力处于非平方区的考虑 ${ }^{[i-6]}$. 第三种看法没有得到阻力试验资料的支持,而 且有的研究者给出的起动流速随水深变化极小‥”。目前很少有人接受. 对于第一、二两种分岐 的存在并不值得奇怪,这是因为细颗起动现象较为复杂、薄膜水附加下压力等机理深入揭示不 够。因而未被晋㴜承认，另一方面考虑附加下压力后文献给出的起动流速受水深的影响太大。 特别是大水深尤其如此、例如当 $d=0.005 \mathrm{~mm}, H=30 \mathrm{~m}$. 分别得出 $V_{K}=6.78 \mathrm{~m} / \mathrm{s}+3.54 \mathrm{~m} / \mathrm{s}$ 及 3. $70 \mathrm{~m} / \mathrm{s}^{i \imath-6]}$, 显然其值过大。不符合室内水槽及野外河道的粘土起动流速一般不超过 $3 \mathrm{~m} / \mathrm{s}$ 的范围泣: 那末是否单颗起动流速公式中考虑水深影响的方次讨高, 根据作者最近分析长江宜 昌站低输沙资料 (相当于起动标准)，水深对起动流速影响很大，当 $h=1-20 \mathrm{~m}$ 时, $V_{K}=f(d) h^{n}$ 中的指数 $n=0.352-0.64$ 这显然不能解释为流速分布的影响 .也不是文献 [1]所能概括的.

其次粘土试验表明，起动时往往不是单颗的，大都是多颗成团进行、特别是干容重较小时 尤其如此。这自然说明多颗成团起动流速小于单颗的. 那末多颗泥沙成团起动时.起动流速如 何确定? 它与单颗起动流速关系如何? 而且, 既然细颗粒往往是多颗成团起动, 说明即令水深 影响大的单颗起动流速公式正确，但是用其确定多颗成团起动流速就不合适了.反之,这有可 能证明单颗起动流速公式未必对大水深不正确, 只是此时不该使用它. 所有这些表明了研究多

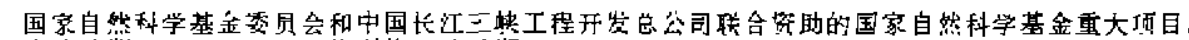

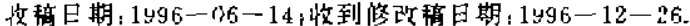

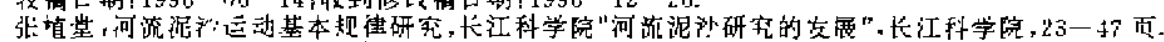

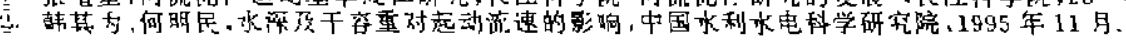


颗泥沙成团起动流速及其单颗起动流速关系重要性. 本文正是从此出发而开展的研究.

为便于与单个细颗粒起动流速等对比, 现将以前研究成果概述如下 ${ }^{[2]}$. 单颗泥沙的䁬时起 动底速为

$$
V_{r-k}=\varphi\left(\Delta^{\prime}\right) \omega_{1}\left(H . d, \gamma_{s}\right)
$$

此处 $\Delta^{\prime}$ 为泥沙在床面的相对位置, $H$ 为水深, $d$ 为单颗泥沙粒径, $\gamma$, 为泥沙比重. 此外

$$
\varphi \Delta\rangle=\left\langle 2 \Delta^{\prime}-\Delta^{\prime 2}\right\rangle+x^{\prime}\left\{\left(\frac{4}{3}-\Delta^{\prime}\right)+\frac{1}{4}\left[\frac{1}{3}+\left(2 \Delta^{\prime}-\Delta^{\prime 2}\right\rangle:\right]\right\}^{\prime}=
$$

而 $\omega_{1}=\left[3.33 \frac{\gamma_{s}-\gamma}{\gamma} g d+\frac{0.0465 \hat{o}_{1}}{d}\left(3-\frac{t}{\delta_{1}}\right)\left(\frac{\delta_{1}{ }^{2}}{t^{2}}-1\right)-1.55 \cdot 10^{-7} \frac{H}{d}\left(1-\frac{t}{\hat{o}_{1}}\right)\left(3-\frac{t}{\hat{o}_{1}}\right)\right]^{\prime}=$

称为起动流速的特征速度. $\gamma$ 为水的比重. $g$ 为重力加速度. $\hat{o}_{1}=4 \times 10^{-7} \mathrm{~m}$ 为薄膜水厚度. 而 $\frac{t}{\hat{o}}$. 为颗粹间的相对缝隙。它决定于㵀积物干容重.即

$$
\gamma_{s}^{\prime}=\left[0.698-0.175\left(\frac{t}{\hat{o}_{1}}\right)^{\frac{1}{3}: t-\frac{1}{\hat{o}_{1}}}\right]\left|\frac{d}{d+2 t}\right|^{3} \gamma_{s}
$$

过渡到以垂线平均速度表示的起动流速, 采用了推移质低输沙率理论关系及起动的数量际准. 得到 ${ }^{[4.8]}$

其中

$$
V_{k}=\psi u_{\cdot . k}=\frac{\psi}{3.73} \bar{V}_{b k}=\frac{0.433}{3.73} \psi \omega_{1}=0.116 \psi \omega_{1}
$$

此处 $V$ 为 (垂线)平均流速, $u$. 动力流速, $u, k$ 相应于起动时的动力流速.

\section{1 细颗粒成团起动的瞬时起动流速}

实际现象表明.具有一定粘性的细颗粒泥沙起动时, 往往不是以单颗、而是以多颗成片 (团) 进行. 开始是小片剥虻, 使具有一定固结的粘土(淤泥)床面形成坑、槽、逐渐形成蜂窝状,

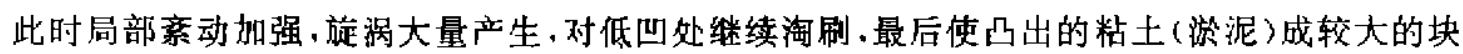
状崩离.经过一定时间滚动.其中较硬者常被磨光成土“卵石”. 河床中常见到的这种土卵石不 仅有的是硬质粘土.而且还有未固结干硬的淤混. 但是从平整床面的冲刷过程看,诱发这种冲 刷“雪崩”的是最早的成片剥蚀. 因此将粘土(湤泥) 的起动. 定义为平整床面最早的成片剥虻. 下面从力学方面对此进行分析.

设在平整的粘土床面上,考虑其中的一土块, 共有 $n$ 个泥沙颗粒组成.它的长和宽为 $u$.厚 为 $c$, 即其扁度

$$
\lambda=a / c
$$

其重量为

$$
\frac{\pi}{6} \gamma_{s} \cdot D_{0}{ }^{3}=\gamma_{1}^{\prime} \cdot c \cdot a^{2}={ }_{n} \gamma_{1} \frac{\pi}{6} d^{3}
$$

其中. $D_{\mathrm{n}}$ 为土块的当量直径 (重量相等的当量直径)， $\gamma_{s}$ 为颗粒比重, $\gamma_{1}^{\prime}$ 为表层土的千容重. 由 (8)式第一等号得

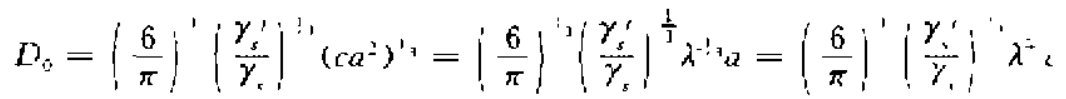

同时由(8)式左、右两端还可给出 


$$
D_{n} / d=n^{\prime}
$$

此六面体在上、下底面积的颗数 $n_{1}$ 由 $u^{2}=n_{1}\lfloor d-$ $2 t)^{2}$ 确定. 即

$$
n_{1}:=u /(d+2 t)
$$

此处 $t$ 为颗粒间缝院的一半 (图 1). 与此相仿六面 体四·个侧面积上的颗粒 $n_{3}$ 由 $\alpha i=n_{2}(d+2 t)=$ 确 定·即

$$
n_{2}{ }^{n}=\frac{(u c)^{2}}{a-2 t}=\frac{u}{d+2 t} \lambda^{-1 *}
$$

此时土块的受力状态如图 2 所示, 不失一般性、图 中示出了 $c=(d-2 t), \alpha=4(d+2 t)$ 的土块.其 上的作用力有水流的升力 $F_{L}$. 床面上的切应力 $\tau_{B}$. 土块的重力 $G$. 底面的粘着力 $F_{k, 1}$ 附加下 压力 $\Delta G$.四周的侧向粘着力 $F_{\mu_{1}}$ 及 $F_{\alpha, 3}$ 将通过摩摬系数 $f$ 转换成铅垂向下的力. 除右侧 $F_{a: 2}$ 外.这些力对于 0 点均有力矩. 至于在单颗泥沙起动中较为重要的水流正面推力, 此时国床面 平整.数量可以忽略.至于其对 0 点之矩更不存在. 欲求出土块起动翻转的条件可列出上述各 力对1)点之矩的平衡方程、

$$
\begin{gathered}
F_{L} \frac{a}{2}+\tau_{b} \frac{l}{2}=F_{m 1} \cdot \frac{a}{2}+F_{m a} f a- \\
F_{n 3} \cdot f \frac{u}{2}+\Delta G \frac{u}{2}-G \frac{a}{2}
\end{gathered}
$$

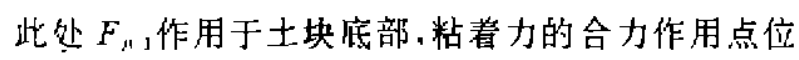
于土块重心，故对 9 点之力锌为 $a / 2 ; F_{m, 2}$ 作用于土 块左侧粘着力的合力. 它通过摩擦系数 $f$ 将其转换 成重力方向的力，其作用点在左侧面重心，力等为 $u: F_{n}$ ，为作用在土块两侧的粘作力的合力，它也要 通过摩擦系数将其转换成与重力方向一致的力, 力 臂则为 $a / 2$. 各力的表达式如下

$$
\begin{aligned}
& F_{S}=\frac{C_{L} \rho}{2} V_{h}{ }^{2} a^{2} \\
& \tau_{b}=\tau_{0} a^{2}=\rho u u^{2} u^{2} \\
& F_{\mu, 1}={ }_{n} P_{n d}=\left\{\frac{u}{d+2 t}\right\}^{2} P_{p, d} \\
& F_{\mu 2}=n_{2} P_{k d}=\frac{1}{\lambda}\left(\frac{a}{d+2 t}\right)^{2} P_{n a} \\
& F_{\mu 3}=2 n_{2} P_{\mu d}=\frac{2}{\dot{\lambda}}\left|\frac{a}{d+2 t}\right|^{2} P_{\mu \omega} \\
& \Delta G=n_{1} \Delta G_{d}=\left|\frac{u}{d+2 t}\right|^{2} \Delta G_{d} \\
& G=\frac{\pi}{6}\left(\gamma_{1}-\gamma\right) D_{0}^{3}
\end{aligned}
$$

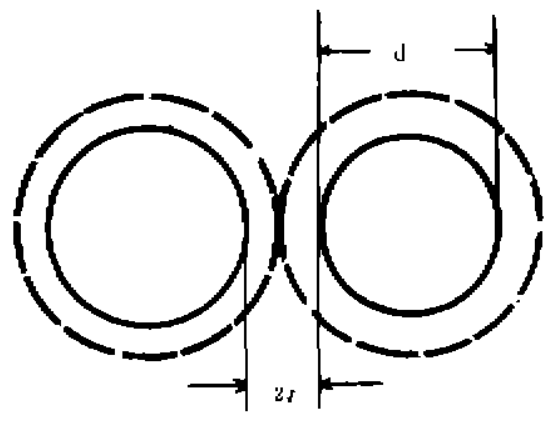

诗1 泥桀颗粒及溥膜水示意图

Fig. 1 Sketch of particle and film water 


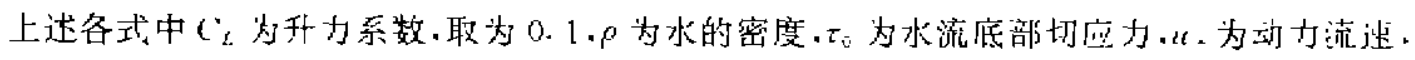

$$
P_{n i}=\frac{\pi}{2} g_{11} \frac{\hat{o}_{12}{ }^{2}}{\delta_{1}^{2}} d\left[\frac{\hat{\delta}_{\mathrm{i}}^{2}}{t^{2}}-1\right]
$$

为两顆泥沙正接触(一颗起动泥沙与正一颗床面泥沙的正接触〉时的枮着力

$$
\Delta \dot{G}_{i}=\frac{\pi}{2} k_{2} \gamma H d \hat{o}_{1}\left(1-\frac{t}{\partial_{1}}\right. \text { ! }
$$

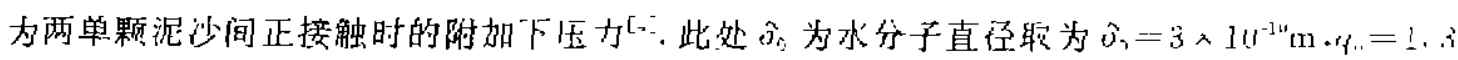
$\times 10^{\circ} \mathrm{kg} / \mathrm{m}^{2} \cdot k_{2}=2.285 \times 10^{-6}$. 将上炢各式代入!11)式.㴚有

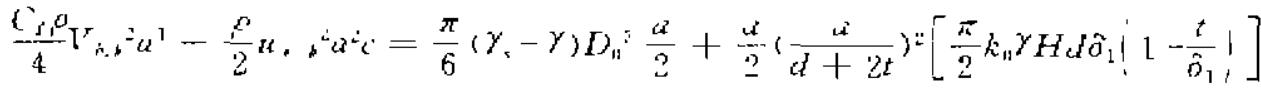

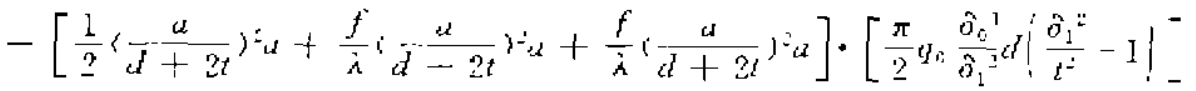

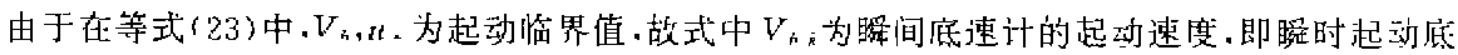

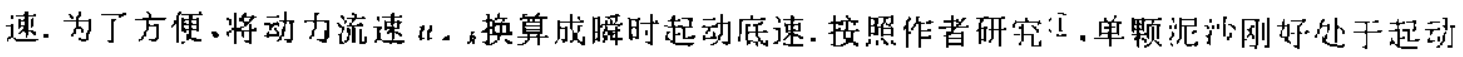
临界状态时, 其瞬时起动流速按(1) 式对床面位犆平均有

$$
V_{i k}=\bar{\varphi} \omega_{1}=0.916 \omega_{1}
$$

另一方面·按照水槽试验起动标准!

$$
\bar{V}_{n}, \ldots 0.433 \omega_{1}
$$

则

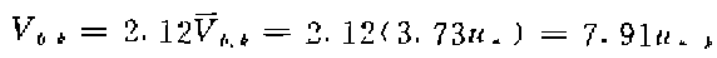

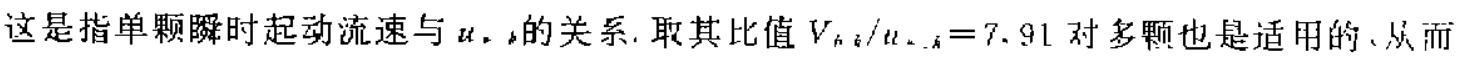
利用此式及(7)式使 (23) 式左端变为

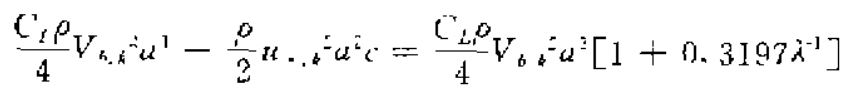

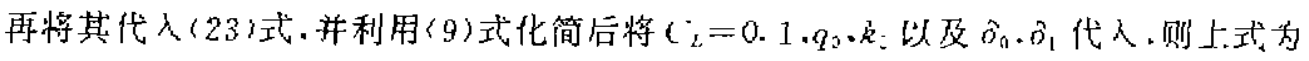

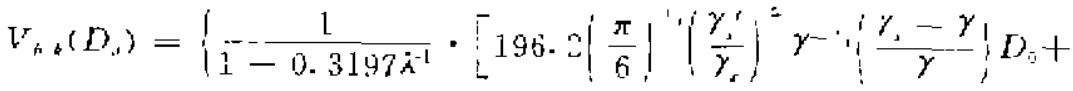

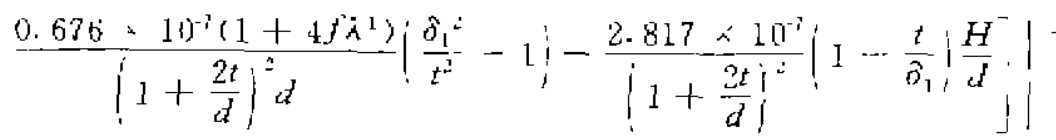

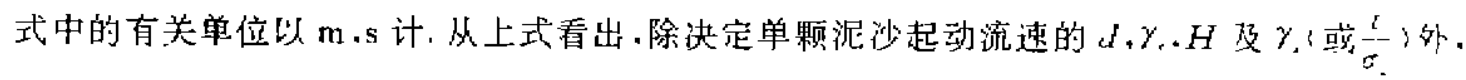

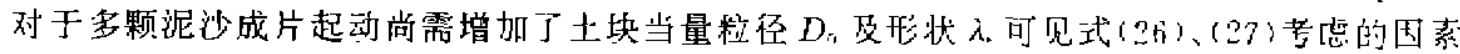
是硕页为全面的.

\section{3 多颗成团起动瞬时底速与单颗的对比}

为了对比方便, 现在仅考虑 $\varphi$ 对 $\Delta^{\prime}$ 的平均值 $\bar{\gamma}=0.916$, 作为泥新在床面位置影响其起䢵 流速 $V_{k, k}$ 这表示研究的单颗泥沙的瞬时起动速度是处于平均位置的值. 对此显然是可以接受

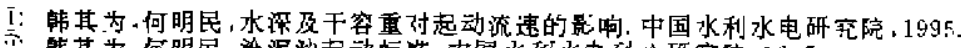

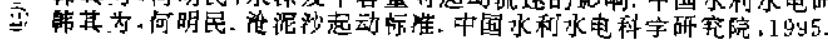


的. 此时( 3 )式代入( 24$)$ 式, 遂有

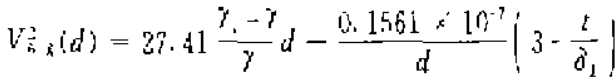

$$
\begin{aligned}
& \left|\frac{\partial_{1}^{2}}{t^{2}}-1\right|+1.361 \cdot 1 r^{-1}\left(1-\frac{t}{\hat{o}_{1}}\right)\left|3-\frac{t}{\delta_{1}}\right| \frac{H}{d}
\end{aligned}
$$

式中的有关单位以 m.s 计、此处以 $V_{b k}(d)$ 表示单颗泥少起动的瞬时底部流速, 为区 别起见用 $V_{k, k}\left(D_{3}\right)$ 表示多颗成片起动的瞬 时底部流速. 现在分析两者的关系. 在图 3 中给出了 $\gamma_{.}=2.65 \mathrm{t} / \mathrm{m}^{2}, d=0.005 \mathrm{~mm}, \frac{t}{\hat{o}_{1}}$ $=U .375$. 单颗起动流速与水深的关系 $V_{k, k}$ (d) $-H$. 与此同时. 在图中同时还给出 $\dot{\lambda}=$ $2 \cdot \frac{t}{c_{1}}=0.375$ 下不同 $D_{0}$ 时, 多颗成片起动 流速 $v_{n, ~}\left(D_{n}\right)-H$ 关系. 从图中可见、每一。 $\left.V_{b}, D_{r}\right)-H$ 曲线与 $V_{k, k}(d)-H$ 曲线均相 交，设交点纳座标为 $H_{3}$ ，当在交点以下，即 $H \leftarrow H_{k}, V_{k, k}\left(D_{1}\right)>V_{h k}(d)$; 当在交点以上 $H>H_{k}, V_{r},\left(D_{a}\right)<V_{k}(d)$; 交点处. $V_{b k}$ $\left(D_{n}\right)=V_{h k}(d)$. 称交点水深为临界水簿 . 即 $V_{k k}\left(D_{0}\right)=V_{n k}\langle d\rangle$ 时的水深. 从图中还 可青出、临界水深随着 $D_{0}$ 的加大而加大. 这是显然的. 需要强调的是水深加大, $V_{h, 4}$ (d)增加快的原因是其薄膜附加下压力占 的比例要大.

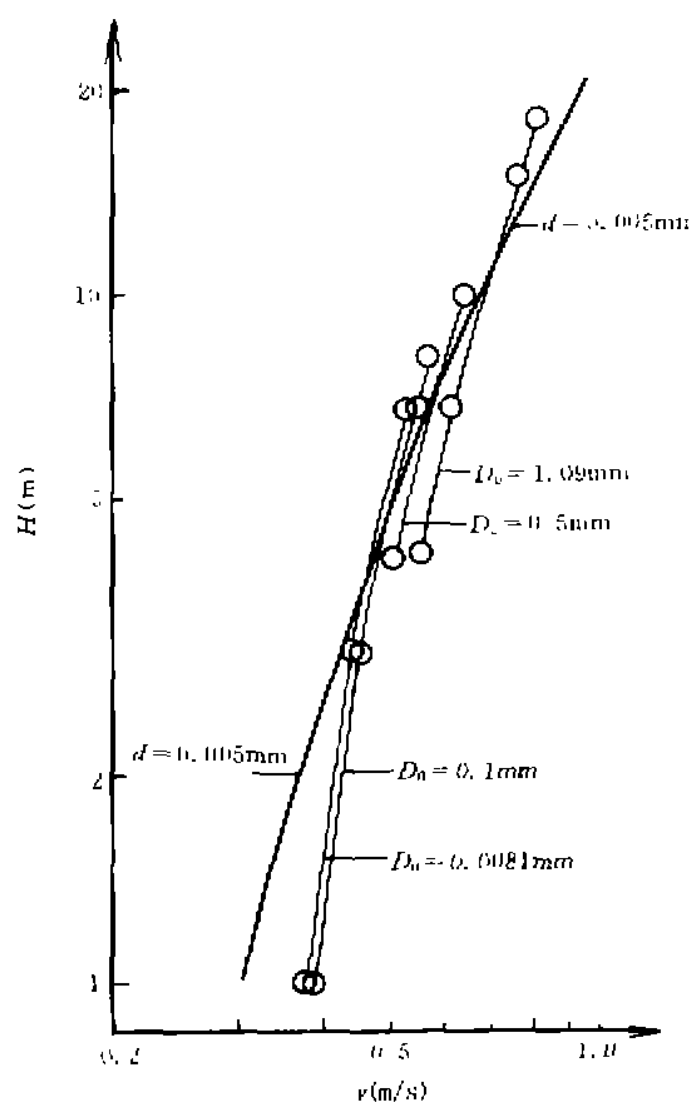

图 3 细颗粹成团起动流速与单颗的对比

Fig. 3 Comparision of incipient motion wirh single particle and aggregated particles

$$
\begin{aligned}
& \text { 令 } V_{b k}\left(D_{1}\right)=V_{k}(d) \text { ，则 } \\
& \left.H_{k}=\left[1.301\left(1-\frac{t}{\hat{\sigma}_{1}}\right)\left(3-\frac{t}{\hat{\delta}_{1}}\right)-2.817\left(1-\frac{t}{\delta_{1}}\right) \cdot \frac{1}{1+0.3197 \hat{A}^{-1}} \cdot 11+\frac{2 t}{d}\right)^{-2}\right]^{-1} \text {. } \\
& \left\{10^{i}\left[196.2 \frac{r_{s}-r}{r}\left\{\frac{\pi}{6}\right\}^{\prime}\left\{\frac{r_{1}^{\prime}}{r_{s}}\right\}^{-i} \lambda^{-2} D_{v} \cdot \frac{1}{1-0.3197 \bar{h}^{-1}}-27.14 \frac{r_{-}-r}{r} d\right] d-\right.
\end{aligned}
$$

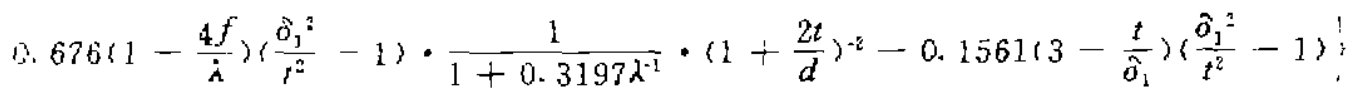

此式的有关单位仍以 $\mathrm{m} . \mathrm{s}$ 计. 现在分析两种特殊情形:

(1) 摩擦系数 $f=0.4 \cdot \gamma_{\mathrm{s}}=2.65 \mathrm{t} / \mathrm{m}^{3}, d=0.005 \mathrm{~mm}, \lambda=2 \cdot \frac{t}{\delta_{1}}=0.375\left(\gamma_{\mathrm{s}}^{\prime}=1.236 \mathrm{t} / \mathrm{m}^{3}\right)$, 即 $t=1.5 \times 10^{-3} \mathrm{~m}$, 此时上式简化为

$$
H_{k}=5437 D_{0}+4.072
$$


(2) $\left.d=0.00 \mathrm{lmm} \cdot \lambda=4 \cdot \frac{t}{\partial_{1}}=0.375 \cdot\left(\gamma^{\prime}=1\right) .67 \mathrm{t} / \mathrm{m}^{3}\right) \cdot t=1.5 \times 10^{-1} \mathrm{~m}$. 此时有

表 1 不同大小土块临界水深

Tab. 1 Critical wates depth for different size lump

\begin{tabular}{|c|c|c|c|}
\hline \multicolumn{2}{|c|}{$d=1)$ bill $1 \mathrm{mmr}$} & \multicolumn{2}{|c|}{$d=1 \% .005 \mathrm{~mm}$} \\
\hline$\Gamma_{4}(\mathrm{~mm})$ & $H_{2}(\mathrm{~m})$ & $D_{11}(\mathrm{~mm})$ & $H_{+}(\mathrm{m})$ \\
\hline 1). 11346 & 0. 5 f. & 11. unk 1 & 412 \\
\hline 11.115 & ก. 584 & 11.05 & 4.34 \\
\hline औ. 1 & 0.611 & a. 1 & 4.62 \\
\hline a. 5 & 11. 732 & (3. 5 & 6.79 \\
\hline 1.0 & 1.. 845 & 1.0 & g. 51 \\
\hline 1. 32 & 1.100 & 1. 119 & 11 nu \\
\hline 2 & 1. 22 & 2 & 14.95 \\
\hline 3 & 1.55 & 3 & 20.34 \\
\hline 5 & 2.21 & 5 & 31.26 \\
\hline
\end{tabular}

$$
H_{k}=327.6 D_{n}+0.568
$$

上述两式的有关量的单位仍以 $\mathrm{m}$ 计.其部分数 字結果如表 1 所示. 表 1 中的临界水深为大小 不同土块瞬时起动底速怡好等于单颗泥沙睬时 起动底速时的水深. 从表中看出对于 $d=$ $0.001 \mathrm{~mm}$ 泥沙. 当水深 $H>1 \mathrm{~m}$ 时. 有 $\Gamma^{\prime}$ ，二 $1.32 \mathrm{~mm}$ 的不同大小土块的瞬时起动流速均小 于单颗的.显然此时已经是土块起动代替丁单 颗的. 与此相仿, 对于 $d=0.005 \mathrm{~mm}$ 泥沙, 当水 深 $H>4.62 \mathrm{~m}$ 或 $H \geqslant 10 \mathrm{~m}$ 时，也有 $\Gamma_{1}<$ $0.1 \mathrm{~mm}$ 或 $D_{0}<1.09 \mathrm{~nm}$ 的大小不同土块起动 代替了单颗起动.

\section{3 片状土块起动时临界水深及起动速度}

实际表明, 粘土土块起动时多为片状. 片状土块最小的厚度应仅为一颗粒、即 $c=1 d+$ $2 t)$ ，此时 $(9)$ 式为

$$
\left.\left.D_{\mathrm{n} m}=\left\{\frac{6}{\pi}\right\}^{\prime} \mid \frac{\gamma_{s}^{\prime}}{\gamma_{\mathrm{r}}}\right)^{\prime} \lambda^{5} d \mid 1+\frac{2 t}{d}\right)
$$

这种土块以下将称为片状土块、其当量粒径记作 $D_{0, m}$. 它的典型组合为 4 -个颗粒组成一片 $1 \dot{\lambda}=$ $2) ; 16$ 个颗粒组成一片 $(\lambda=4)$ 等. 将(33) 式代人 $(30)$ 式, 并考虑天然紧取 $\gamma_{s}=2.65 \mathrm{t} / \mathrm{m}$. 遂有

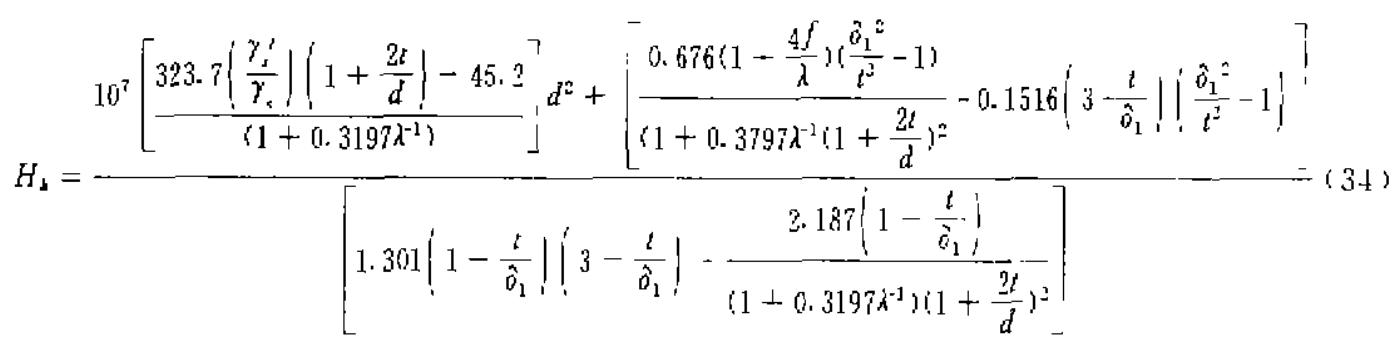

此式的有关单位仍以 $m+s$ 计, 在表 2 中列 出了 $\lambda=2 \cdot \frac{t}{\delta_{1}}=0.375$, 即 $t=1.5 \times 10^{-7} \mathrm{~m}$. 不同粹径泥沙的片状土块的 $\gamma^{\prime}, D_{0 . \mathrm{m}}$ 及临 界水深 $H_{k}$. 从表中看出临界水深随单颗泥 沙的粒径加大而增加. 在常规干容重 $\left(\frac{t}{\delta_{1}}=\right.$ $0.375)$ 下. 有下述结果: 1 ) 对于粘土 $(d<$ 0. $005 \mathrm{~mm}) . H>4.12 \mathrm{~m}$ 时: 23 对于细粉土 (1). $005-0.010 \mathrm{~mm}) . H>5.18 \mathrm{~m}$ 时；3) 对 于粗粉土 $(0.01-0.05 \mathrm{~mm}), H>10.30 \mathrm{~m}$
表 2 不同粗细泥沙片状土块起动临界水涴

\begin{tabular}{|c|c|c|c|c|}
\hline$d(\mathrm{~mm})$ & $\left(1+\frac{2 t}{d}\right)$ & $\gamma_{i}:\left\{t \mathbf{m}^{3}\right\}$ & $\Gamma_{c m}(\mathrm{~mm})$ & $H_{+}\{\mathrm{m}$ I \\
\hline $0.0 n 1$ & 1.30 & ก. ค่ & 11. vr 2612 & 1. : it \\
\hline 0.004 & 1.175 & 1.185 & ii. 010648 & 373 \\
\hline i. 005 & 1. 66 & 1.236 & $1 \% \pi 1181$ & 4. 12 \\
\hline 0.01 & 1.03 & 1.347 & 0.0162 & 5.18 \\
\hline 1). 115 & 1. 0116 & 1. 446 & 11.1181 & 16.30 \\
\hline
\end{tabular}

Tab. 2 Critical water depth of incipient motion for layared soil with different particle size 
时。几乎均以多颗成片起动进行.

表 3 不同千容重.不同扁度、片状土块起动临界水㺷

Tab. 3 Critical water depth of inciplent motion for layered soil withdifferent dry density and humf ellpricity

\begin{tabular}{|c|c|c|c|c|c|c|c|c|}
\hline \multirow{2}{*}{$\frac{t}{b_{1}}$} & \multirow{2}{*}{$\left(1-\frac{\pi t}{t}\right)$} & \multirow{2}{*}{$\begin{array}{c}7{ }^{\prime} \\
\left(1 / \mathrm{m}^{\prime}\right)\end{array}$} & \multicolumn{2}{|c|}{$\lambda=\mathbf{I}$} & \multicolumn{2}{|c|}{$\lambda=4$} & \multicolumn{2}{|c|}{$A=2^{\prime \prime \prime}$} \\
\hline & & & $\Gamma_{1, m}(\mathrm{~mm})$ & $H_{k}(\mathrm{~m})$ & $\left.\Gamma_{1}\right]_{m}(\mathrm{~mm})$ & $H_{4}(\mathrm{~m})$ & $\Gamma_{n}$ immi & $\mid f,\{\mathrm{~m} \mid$ \\
\hline :1. 2011 & $1 .: 132$ & 1.408 & n. 01$) 23$ & 12.10 & 0.0131 & $9.7^{2}$ & 11. U13ร2 & li. +1 \\
\hline$\% 735$ & 1.075 & 1. 236 & 0.00810 & 4.12 & c. 0128 & 3.35 & 11. 11376 & $2 .+2$ \\
\hline II. זחו" & 1.11811 & 1.140 & 0. 6108173 & 2.65 & 0.0127 & 2.18 & 11. 11372 & 1.61 \\
\hline $11.80 \% 11$ & 1.128 & $11.97 \mathrm{u}$ & 11.100795 & 1. 51 & (1. & 1. 32 & ก. 11369 & 1.117 \\
\hline
\end{tabular}

为研究扁度 $\lambda$ 和干容重对成块起动的临界水深的影响. 按照(34)式计算了 $d=0.1005 \mathrm{~mm}$. $\lambda-2.4 .20$ 及 $\frac{t}{\delta_{1}}=0.2,0.375 .0 .500 .0 .8$ 等各条件下的 $H_{k}$. 如表 3 所示. 可以看出: 当单颗沉

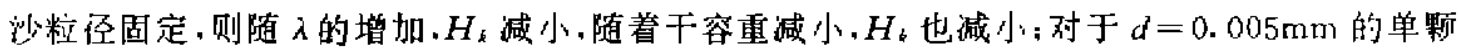
泥渷, 当干容重较小时. 临界水深可降至 $1 \mathrm{~m}$ 左右. 可见在天然河道.特别在水库细颗粒游积 处.当泥沙起动时多颗成片进行是较为普遍的形式.

在表(4)中按照 (34)式计算了 $d$ $=0.001 \mathrm{~m}, \lambda=4$ 不同干容重泥沙起动 时的临界水深. 可见对于这种泥沙.当 干容重很小时.片状土颗的临界水深 很小, 几乎为零. 当然 $H_{k}$ 为负值的物 理意义是对于任何水深.单颗起动流 速不可能小于多颗片状土块的起动流 速. 换句话说, 当 $d=0.001 \mathrm{~mm}$, 且干 容重很小时.单颗泥沙起动几乎不存 在.均是多颗成片进行.
表 $4 d=0$. $001 \mathrm{~mm}$ 时片状土块起际临界水深 Tab. 4 Critical water depth of incipient motion for layered soil with $d=0$. $0101 \mathrm{~mm}$

\begin{tabular}{|c|c|c|c|c|}
\hline \multirow{2}{*}{$-\frac{t}{\partial_{3}}$} & \multirow{2}{*}{$1+r 8 \frac{t}{\partial_{1}}$} & \multirow{2}{*}{$\begin{array}{c}\gamma_{s}^{\prime} \\
\left(t \mathrm{~m}^{\prime}\right)\end{array}$} & \multicolumn{2}{|c|}{$\lambda=4$} \\
\hline & & & $n z_{m}(\min )$ & $\left.H_{\mathrm{a}} \mid \mathrm{m}\right)$ \\
\hline 1. 200 & 1.16 & ก. 992 & 11. $19040=$ & $\therefore 7 t$ \\
\hline ט. 375 & 1.30 & 11.674 & 17. 14396 & $115 e^{\prime \prime}$ \\
\hline 11. 5no & 1. $40 ;$ & 17. 524 & a. 1311393 & 1. 1 in \\
\hline 11.710 & 1. 56 & 0. 369 & (3. r) r)1389 & 0. $025 \div$ \\
\hline (1. 8140 & 1. 64 & 0. 316 & 0. & - (i. 0158 \\
\hline
\end{tabular}

\section{4 片状土块起动时垂线平均流速与单颗的对比}

在表 5 中列出了单颗泥沙 $d=0.005 \mathrm{~mm}, \frac{t}{\delta_{2}}=0.2 .0 .375 \cdot 0.500 .0 .8 . \lambda=4$ 在不同水深条 件下按上述有关公式计算的片犾土颗瞬时起动底速 $V_{b .4}\left(D_{0}\right)$ 和垂线平均起动底速 $V_{k}\left(D_{a}\right)$. 及 单颗瞬时起动底速 $V_{b, k}(d)$ 和垂线平均起动流速 $V_{b}(d)$. 其中 $V_{b, k}\left(D_{0}\right)$ 按 (28)式计算. $V_{b} b(d)$ 按 (29)计算, $V_{k}(d)$ 按 $(5) 、(3)$ 式计算. 而 $V_{\boldsymbol{1}}(D)$ 由于暂时未将 $V_{b, d}\left(D_{0}\right)$ 和低输沙率联系起来. 难以直接给出计算的公式, 但可以按 $V_{n k}(d)$ 与 $V_{k}(d)$ 的比值进行, 即

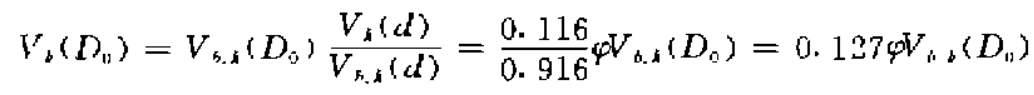

从表 5 可见：(1) 以起动临界水深为界, 当 $H<H_{k}$ 时. $V_{k}\left(D_{0}\right)>V_{k}(d)$ ）当 $H>H_{b}$ 时. $V_{\text {， }}$ $(D),\left\langle V_{b}(d)\right.$; 而当 $V_{b}\left(D_{i}\right)=V_{b}(d)$ 时, 则 $H=H_{k}$. 这一点与瞬时起动底速规律一致. (2) 在表 中所示 $H>K_{b}$ 的范围. 片状土块起动流速 $V_{b}\left(D_{0}\right)$ 仅为 $V_{b}(d)$ 的 $80 \%-90 \%$. (3) 如以 $d=$ 
$0.005 \mathrm{~mm}$ 代表粘土.而 $\frac{t}{\delta_{1}}=0$. （相应的 $\gamma_{\mathrm{r}}=1.408 \mathrm{t} / \mathrm{m}^{3}$ ) 为密然情况, 则当水深在 $10 \mathrm{~m}$ 以时.片 状土块起动流速一般在 $3 \mathrm{~m}$ 以下.或在 $3 \mathrm{~m}$ 左右. 这与室内试验与野外测量数据一致.

表 5 片将土块垂线平均起动流速与单颗对汇

Tab. 5 Cnmparsion of incupent velocity averaged of single partucle in verrical direction with luytrcal-ul

\begin{tabular}{|c|c|c|c|c|c|c|c|c|c|}
\hline$\frac{i}{n_{1}}$ & $\begin{array}{l}E S \\
\{m !\end{array}$ & $\begin{array}{c}y_{1}^{\prime} \\
\text { itim: } 1\end{array}$ & $\begin{array}{c}\Gamma \mathrm{i}_{11} \\
i \mathrm{~mm} !\end{array}$ & $\begin{array}{c}L_{h},\left(D_{i}\right) \\
(\mathrm{m} / \mathrm{s})\end{array}$ & 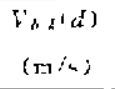 & $\varphi$ & $6 m_{11}<1$ & 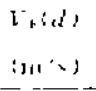 & $\begin{array}{l}{[\because, i, 1} \\
\vdots \pi+\cdots\end{array}$ \\
\hline i. 2 & B. $(81)$ & 1. 413 & 11. : :31 & $\Leftrightarrow 74-1$ & n. 748 & 25.46 & il & 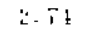 & $\therefore \cdot n i$ \\
\hline & 1. & & & $\therefore 481$ & 11. 861 & 26.55 & 11. $45: 2$ & $\therefore 4 f_{1}$ & 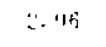 \\
\hline & 1: & & & U. 531 & 1. 45.3 & 26 . if & 1.1141 & $\therefore 2 i$ & .. Il \\
\hline- & $\ddot{2}$ & & & 1.1186 & 1.173 & 27.27 & 1. $281:$ & 4.115 & $\therefore=$ \\
\hline 11.375 & $1.4 t^{\prime}$ & 1231 & (1) $1: 129$ & 11. 354 & a. 3115 & 24.15 & 11.533 & 1.. $4: 3$ & . in \\
\hline & 3340 & & & ก. $4+411$ & 0.440 & 25.45 & 0. $4 \mathrm{kO}$ & 1.42 & $1 \dddot{2}$ \\
\hline & iे $\| 11$ & & & 11. 5:"ll & i. 554 & 26.06 & ง. 5114 & $1.8 s$ & 17 \\
\hline & $9.1 ! 11$ & & & "1 598 & (1. 65t & 26.48 & n. 719 & $\because .21$ & $\therefore 1: 1$ \\
\hline & 15.110 & & & a. 729 & 0. 831 & 26.99 & 0 yin & 284 & $\therefore 44$ \\
\hline \multirow[t]{4}{*}{ (1. ร)แ } & a. $5 n$ & $1.14 ! 1$ & 11.1127 & 7). 258 & 1.. 200 & 43.36 & 0.218 & (i. 51! & it. $71:=$ \\
\hline & 2.177 & & & 0.3117 & ก. 307 & 25.1311 & (1. 335 & 1. 973 & 11.1 .75 \\
\hline & 4. 10 & & & 0.368 & 10. 35: & 25.614 & 11.427 & 1.27 & 1.14 \\
\hline & H. (ir) & & & $0.52-1$ & $\pi .533$ & 29.36 & 0.582 & 1.78 & 1. $4:$ \\
\hline \multirow[t]{5}{*}{$11.81: 1$} & 0.5 & n. 070 & 11.1126 & $\therefore 112$ & $0 . n 49$ & 23.36 & 11.108 & ก. 293 & ii. 311 \\
\hline & 1.516 & & & 11.138 & n. 138 & 24.45 & n. 151 & 11. 428 & (1) $1: 8$ \\
\hline & 210 & & & 11.182 & n. 192 & $25 \cdot 34$ & 1). 214 & 13. $62 y$ & 1. 584 \\
\hline & $8.11 \%$ & & & 11. 2441 & (2. 3119 & 26.36 & 11.333 & $1.1: 5$ & 1. R(Ii) \\
\hline & 12. :111 & & & 1. 326 & 0.326 & 26.76 & 13.441 & 1.27 & 1. 111 \\
\hline
\end{tabular}

\section{5 成块起动流速的验证}

细颗粒成块(片)起动的试验筫料较少,而且由于起动后相当一部分颗粒转为悬涼, 体承流 浑浊, 观测起来也颇为困难. 此处仅搜集到黄岁梁. 陈稚喤等人利用塑料沙做的试验

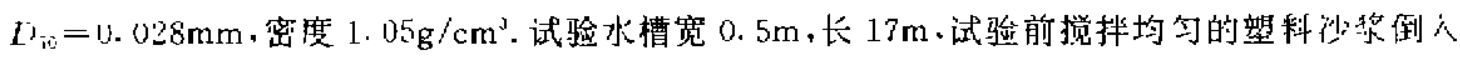
槽中,静置沉积、以形成䏆内 $5 \mathrm{~cm}$ 厚的沙层.然后上稪清水,待水下固结. 水下固结试验苦进行 3 次、第一次固结 $12 \mathrm{~d}\left(\right.$ 相应干容重 $\left.0.603 \mathrm{~g} / \mathrm{cm}^{3}\right)$. 第二次固结 $125 \mathrm{~d}$ (相应干容重 $0.648 \mathrm{~g} / \mathrm{cm}^{2}$ )。 第三次固结 $185 \mathrm{~d}$ (相应干容重 $0.659 \mathrm{~g} / \mathrm{cm}^{3}$ )，每次固结后・放三种水深做起动流速试验。试验中 观察到“当水下固结 $12 \mathrm{~d}$ 时,塑料沙是呈现一丝丝、一缕缕的起动破坏. 水下固结时间增加时。 如固结 185 天时.塑料䏚呈现一小团、一小块的起动破坏"[1] . 因此可以认为在本项试验中，细 颗粒起动时, 处于单颗与成团起动的临界状态.或者说两者都能起动. 当固结时间短时.以单颗 起动为主、从而出现一丝丝、一缕缕的破坏，而当固结时间长时，则开始有成团、成块起动. 当然 此时也会有单颗起动，只是目标不如成团、成块明显、观察时不易醒目罢了.

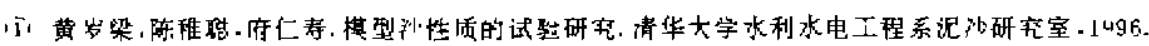


表 5 成垬起动流速检验

Tab. 6 Vexifıcation of incipıent velocrty of layered sol

\begin{tabular}{|c|c|c|c|c|c|c|c|c|c|}
\hline \multirow[b]{2}{*}{$\begin{array}{c}H \\
: \\
: 1:\end{array}$} & \multicolumn{2}{|c|}{ 原蛇据 } & \multirow[b]{2}{*}{$\frac{t}{\partial_{1}}$} & \multirow[b]{2}{*}{$\psi$} & \multirow{2}{*}{ 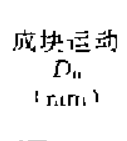 } & \multicolumn{2}{|c|}{ 成塻语动 } & \multicolumn{2}{|c|}{ 单野起动 } \\
\hline & $v_{t}$ & $\begin{array}{c}\gamma_{1} \\
\left(1 / m^{\prime}\right)\end{array}$ & & & & $\begin{array}{c}V_{0},\left(\Gamma_{0}\right) \\
{\left[m_{s} s\right)}\end{array}$ & $\begin{array}{l}\left(1,\left\{\Gamma_{10}\right)\right. \\
(m)\end{array}$ & 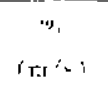 & 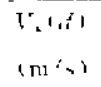 \\
\hline 1.1 & 13.173 & $\therefore 5113$ & 11. $21:$ & 1 R. 07 & a. $31 x$ & 1i. (1]4 & (i. 11626 & is & (1. A \\
\hline 1.1 & $\therefore 1: \frac{1}{4}$ & 9.548 & $\therefore 1: 72$ & 18.9 & :1. $x \leq 7$ & 11. $0,5,5\rangle$ & $\Rightarrow 1 \hat{2} b$ & 11. 17117 & i. L1ut: \\
\hline 1.4 & $\therefore 15.2$ & $\therefore 659$ & $1.1 i_{i j}$ & 18.117 & 11. 338 & 0.0354 & (1. 173 & 11.5681 & "1. 1. \\
\hline 7.5 & $11.118 \mathrm{t}$ & 1. 6.17 .2 & 1). In & 18.81 & 11,300 & 0. 17324 & 11. 17774 & 11. 1346 & is bistiti \\
\hline 7.7 & 11.143 & 11.648 & 11.1172 & 18.81 & 0. 3.37 & 0.0575 & 11.137 & 11.531 & $\because 1: 1:$ \\
\hline 73 & 1135 & $\overline{0} .5 \overline{5} ! !$ & 11,215 & $18 \cdot 81$ & ค. 338 & (1. 0773 & ก. 185 & 1). 0715 & D. $15 . t i$ \\
\hline 11.5 & त 1115 & 0). $5: 13$ & $0.2 n$ & 19.34 & 1). 300 & 11. 0366 & 0.01904 & 0.11412 & 1. Mato \\
\hline 11.5 & 0.15 .5 & C. $\hat{4} 48$ & 6. 1:272 & 19. 3! & 0.337 & 1. תIGLF & r 137 & 0.6113 & 1. 134 \\
\hline 115 & (j) 193 & (1. 659 & $v .115$ & 19.30 & i1. .338 & ก. 11345 & (i. 196 & ניתו1י & $: 1.174$ \\
\hline
\end{tabular}

试验的9 个资料如表 6 所示. 由于是塑料沙.粘着力很小.按作者验证.塑料悊.其拈着力 仅为天然沙的 $\frac{1}{200}$. 因此对片状土块, 将式(33)代入式(28)起动有底速为

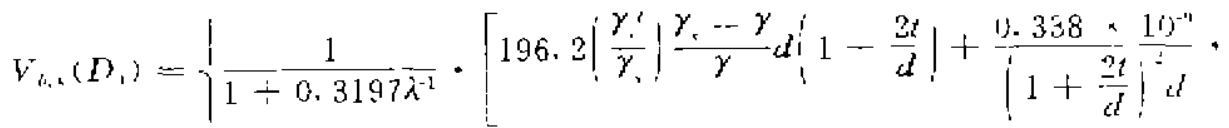

$$
\begin{aligned}
& \left\langle 1+4 \frac{f}{\lambda},\left\{\frac{\partial_{1}^{2}}{t^{2}}-1 \mid+\frac{2.817 \times 10^{-7}}{\left\{1+\frac{2 t}{d}\right)^{2}}\left(1-\frac{t}{\partial_{1}}\right)\left(\frac{H^{-}}{d}\right)\right\}\right.
\end{aligned}
$$

对 $F d=0.028 \mathrm{~mm} . \gamma=1.05 \mathrm{t} / \mathrm{m}^{3} \cdot \lambda$ 取为 20 的片状土块. 则

$$
\begin{aligned}
& V_{h, \lambda}\left(D_{n}\right)=2.704 \times 10^{-4} \frac{\gamma_{r}^{\prime}}{\gamma_{2}}\left|1+\frac{2 t}{d}\right|+\frac{0.1283 \times 10^{-4}}{\left|1+\frac{2 t}{d}\right|^{2}}\left\{\frac{o_{1}^{2}}{t^{t}}-1\right\}+ \\
& 99.0 \times 1 u^{-4}\left(1-\frac{t}{\delta_{1}} \mid \frac{H}{\left\{1+\frac{2 t}{d}\right)^{2}}\right]^{2}
\end{aligned}
$$

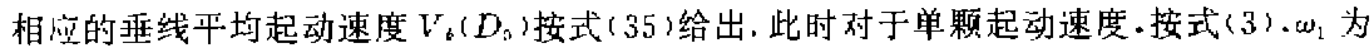

$$
\omega_{3}=\left[0.4533 \times 10^{-4}+3.321 \times 10^{-6}\left\{3-\frac{t}{\partial_{1}}\right\}\left\{\frac{\partial_{1}^{2}}{t^{2}}-1\right\}+5.535 \times 10^{-5} \mathrm{H}\left\{1-\frac{t}{\partial_{1}}|j| 3-\frac{t}{\partial_{1}}\right\}\right]^{\prime 2}
$$

其中已将 $d=0.028 \mathrm{~mm}$ 代入. 此式及式 (37)中有关单位均取 $\mathrm{m} . \mathrm{s}$ 、至于以垂线平均流速表示 的单颗起动速度 $V_{k}(d)$ 与瞬时起动底速的关系前面已指出按式 (5) 给出. 这样计算的单颗起动 流速的 $\omega_{1} 、 V_{k}(d)$ 及多颗成片起动的 $V_{n k}\left(D_{0}\right)$ 及 $V_{b}\left(D_{n 1}\right)$ 亦列如表 6 中. 从表中看出. 对于 9 次 试验资料. 计算的单颗起动流速均小于实测流速, 但差别不大. 可以认为尚处于起动. 而片状士 块水深为 $0.044 \mathrm{~m}$ 时.起䢵流速大于实测的, 而当水深为 $0.075 \mathrm{~m}$ 及 $0.115 \mathrm{~m}$ 时.两者相近. 综 客起来看. 可以认为在试验中水流速度已达到单颗起动流速. 故总是有单颗泥沙起动. 而对于 片状土块. 当水深为 $0.044 \mathrm{~m}$ 时, 成片起动流速略高于实际流速, 故成片起动少: 当水深为 0. $075 \mathrm{~m}$ 及 $0.115 \mathrm{~m}$ 时. 成片起动流速与实际流速接近, 故有土块起动. 尚需说明的是. 表 6 中 
的片状土块其当量直经为 $0.3-0.338 \mathrm{~mm}$. 而成片的面积为 $(0.563 \mathrm{~mm})^{2}-(0.561 \mathrm{~mm})^{2}$ ，即机 当于 400 个 $0.028 \mathrm{~mm}$ 的泥沙排成一块.每边 20 颗.而厚度则为一个颗粒厚 $d(1-2 t)$. 由此可 见, 成片起动时目标是较大的, 容易观察到.

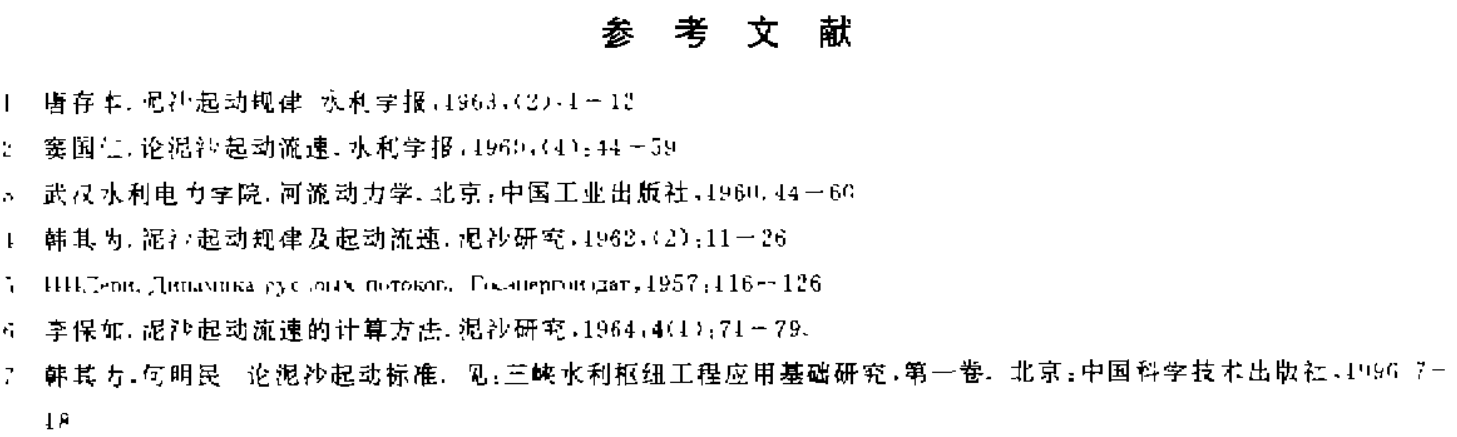

\title{
THE INCIPIENT MOTION AND VELOCITY OF AGGEGATED FINE PARTICLES
}

\author{
Hang Qiwei He Mingmın

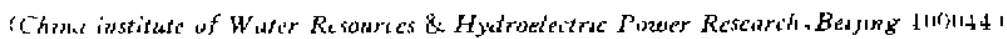

\begin{abstract}
Linder deep water, the incipient velocity calculated from the formulae for single fone particle under cohesive force and additional pressure of film water is much larger than real one and even more than $3 \mathrm{~m} / \mathrm{s}$ ju some cases. Besides, in the nature. sometimes. thefine sediment is aggragated as soll lump or layered soul, nut not in single partscle to start moving. In this paper. the critical condition of nucipient motion for the aggregated soil under cuhesive force and additional pressure of film water is studied, and the incipient velocity is derived. The critical depth $H_{k}$ is introduced after comparing the incipient motion of single particle with that of lump and layered soil, In the cases of $H$ (water depth) $>H_{k}, H=H_{k}$ or $H=H_{4}$. the incipient velocity for single particle may be greater than. equal to or less than that of aggregated soil, respectively. When $H>H_{k}$, the nncipient soil is mainly in aggregated structure. The calculation reveals that $H_{k}$ reduces with the decrease of $D_{2}$, the size of soil lump. dry density and single particle size, and with the increase of lump ellipttcty under certaun conditions. In some cases, $H_{*}$ may be very small and even zero. so that in mespient motion. the sediment will be aggregated in lump or layered soil under any water depth. The formulae for aggregated fine particles have been verified in laboratory, and show good agreement with the observed data.
\end{abstract}

Key Words Incipient motion of lump. fine particle . criticle depth. layered soil 